\title{
On the nodal structure of atomic eigenfunctions
}

\author{
M Hoffmann-Ostenhof $\uparrow \ddagger, T$ Hoffmann-Ostenhof $\S$ and B Simon $\| \uparrow$ \\ $\uparrow$ Institut für Theoretische Physik, Universität Wien, Boltzmanng. 5, A-1090 Wien, Austria \\ $\S$ Institut für Theoretische Chemie und Strahlenchemie, Universität Wien, Währingerstr. \\ 17, A-1090 Wien, Austria \\ || Department of Mathematics and Physics, Princeton University, Princeton, NJ 08544 , \\ USA
}

Received 20 July 1979

\begin{abstract}
We show that in an arbitrarily small neighbourhood of any zero of a real-valued bounded continuous atomic wavefunction, the wavefunction has both signs.
\end{abstract}

Very little is rigorously known about the structure of the set of zeros of a bound state wavefunction for a non-separable quantum-mechanical problem. Atomic wavefunctions are known to be real analytic away from coincidence points (points with either $\boldsymbol{r}_{i}=0$ or $\boldsymbol{r}_{i}-\boldsymbol{r}_{j}=0$ ), but this says surprisingly little about the zero structure. In this paper, we announce and discuss the proof of a result which will be presented more fully elsewhere (Hoffmann-Ostenhof M, Hoffmann-Ostenhof T and Simon B 1980, unpublished).

Theorem. Let $u$ be a real-valued bounded continuous function of $n$ three-vectors obeying

$$
\left[-\frac{1}{2} \sum_{i=1}^{n} \Delta_{i}-\sum_{i=1}^{n} Z\left|\boldsymbol{r}_{i}\right|^{-1}+\sum_{i<j}^{n}\left|\boldsymbol{r}_{i}-\boldsymbol{r}_{j}\right|^{-1}\right] u=\lambda u
$$

Suppose that for some $x_{0} \in \mathbb{R}^{3 n}, u\left(x_{0}\right)=0$. Then $u$ has both signs in an arbitrarily small neighbourhood of $x_{0}$.

\section{Remarks.}

(i) It is merely for convenience that we consider only atomic wavefunctions. The same result holds for molecular wavefunctions in general or in the clamped nuclear approximation.

(ii) Since the zero set must separate two open sets, this theorem says that, in some sense, the zero set must be $(3 n-1)$ dimensional.

(iii) It is a result of Kato (1957) (see also Reed and Simon 1978) that square integrable eigenfunctions are automatically bounded and Hölder continuous, so the theorem applies to them. It also applies to suitable 'continuum' eigenfunctions. 
(iv) The Coulomb nature of the potentials is irrelevant for the truth of the theorem. So long as the two-body potentials $V_{i j}$ obey

$$
\sup _{x} \int_{|x-y| \leqslant 1}\left|V_{i j}(y)\right|^{3 / 2+\epsilon} \mathrm{d}^{3} y<\infty
$$

for some $\epsilon>0$, one can conclude that either $u$ changes sign arbitrarily near $x_{0}$ or $u$ is identically zero near $x_{0}$. The latter possibility can be ruled out by unique continuation theorems in some cases (Reed and Simon 1978, Simon 1979) and in particular in the case of Coulomb potentials (Reed and Simon 1978).

(v) Some restrictions on the potential are clearly necessary for a result of the type we describe; for suppose, in the three-dimensional case, $V$ is spherically symmetric and $V(r) \sim a r^{-2}$ for $r$ small. Then spherically symmetric eigenfunctions will vanish as $r^{b}$; $\left\{b=\frac{1}{2}\left[-1+(1+4 a)^{1 / 2}\right]\right\}$ at $r=0$ and, in particular, $r=0$ will be an isolated zero.

(vi) The ground state $u_{0}(x)$ without statistics (in particular, the true helium ground state) is known to be almost everywhere positive (see Reed and Simon 1978). This theorem then implies that $u_{0}(x)>0$ for $|x|<\infty$. This last result is not new: it was obtained in Simon (1979) by mildly extending some ideas of Carmona $(1978,1979)$. One of our proofs described below is motivated by Carmona's ideas.

We have two different proofs of the above theorem. The first uses Harnack-type inequalities, i.e. if $u$ is a solution of (1) which is non-negative in some bounded domain $\Omega$, then for any strictly contained subdomain $\Omega^{\prime}$ of $\Omega$

$$
\inf _{x \in \Omega^{\prime}} u(x) \geqslant C \sup _{x \in \Omega^{\prime}} u(x)
$$

where $C>0$ depends only on $\Omega, \Omega^{\prime}, \lambda$ and $Z$. There is an extensive literature on such inequalities reviewed in the book of Gilbarg and Trudinger (1977). Essentially the following is proven there. Suppose $u$ is a function non-negative, continuous and bounded in some $\Omega \subset \mathbb{R}^{m}(m \geqslant 3)$ satisfying $(-\Delta+W) u=0$, then (3) holds, provided $W$ obeys (2) (with 3 replaced by $m$ ). This amounts to requiring $W \in L^{m / 2+\epsilon}(\Omega)$ and hence does not allow potentials of the type in (1). However, in an earlier paper in a somewhat hidden remark Trudinger $(1973 \S 5$, remark 1$)$ states that such a $L^{p}$-condition can be replaced by some form boundedness condition. Indeed his proof of (3) extends easily to that case with minor modifications. By standard arguments (Reed and Simon 1978) this form boundedness condition can be shown to hold for sums of two-body potentials satisfying (2).

The second proof which, as we mentioned, is motivated, in part, by the work of Carmona (1978) uses a Feynman-Kac formula:

$$
u\left(x_{0}\right)=\mathrm{e}^{t \lambda} E\left\{\exp \left[-\int_{0}^{t} V\left(x_{0}+b(s)\right) \mathrm{d} s\right] u\left[x_{0}+b(t)\right]\right\} .
$$

In (4), $b$ is Brownian motion, and $E$ is an expectation with respect to it; see Simon (1979) for a discussion of Brownian motion and applications to quantum mechanics. Carmona used (4) to prove that if $u$ is non-negative and not identically zero, then $u\left(x_{0}\right)>0$ as follows: a non-trivial subset of the Brownian paths $\left(x_{0}+b(s)\right)_{s \leqslant t}$ will end at points $y=x_{0}+b(t)$ where $u(y)>0$, so these paths give a strictly positive contribution to the right-hand side of (4); the other paths give a non-negative contribution so $u\left(x_{0}\right)>0$. We modify his argument to prove our theorem, as follows: if $u\left(x_{0}\right)=0$ but $u$ does not have both signs near $x_{0}$, we can suppose $u$ is non-negative in a ball $B=\left\{x:\left|x-x_{0}\right|<r\right\}$ about $x_{0}$. By unique continuation, $u$ is strictly positive somewhere in the ball. For very 
small times, $t$, the overwhelming bulk of the paths in (4) will end up inside $B$, so one can show that the contribution of these paths dominates in absolute value the contributions of paths that end outside $B$. But the contribution of the paths ending in $B$ is strictly positive, so the sum of the two kinds of contributions is positive.

One of us (BS) would like to thank Professor W Thirring for the hospitality of the Institute for Theoretical Physics of the University of Vienna where this work was done.

\section{References}

Carmona R 1978 Commun. Math. Phys, 62 97-106

1979 J. Func. Anal. to appear

Gilbarg D and Trudinger N S 1977 Elliptic Partial Differential Equations of Second Order (Berlin: Springer-Verlag)

Kato T 1957 Comm. Pure Appl. Math. $10151-77$

Reed M and Simon B 1975 Methods of Modern Mathematical Physics, II. Fourier Analysis, Self-Adjointness (New York: Academic)

- 1978 Methods of Modern Mathematical Physics, IV. Analysis of Operators (New York: Academic) Simon B 1979 Functional Integration and Quantum Physics (New York: Academic)

Trudinger N S 1973 Ann. Scuola Norm. Sup. Pisa (3) 27 265-308 